\title{
On Essence of Physical Exercise Habit and Sports Fitness Belief Model
}

\author{
Dehou Ma \\ Xi'an Peihua University, Xi'an, Shaanxi 710125, China
}

Keywords: Physical exercise habit, Essence, Formation mechanism, Sports fitness, Belief model.

\begin{abstract}
Training physical exercise habit of college students and laying a solid foundation for their lifelong physical exercise are the key to college physical education. Students' physical exercise habit directly influences the formation of students' sports fitness belief. The formation of college students' physical exercise habit needs to be based on multiple mechanisms and psychology. Thus, it is necessary to enhance training of students' physical exercise habit. This paper mainly combines actual conditions to analyze the essence of college students' physical exercise habit and formation mechanism, and then discusses the theoretical model of sports fitness belief, in the hope of bettering promoting college students to form physical exercise habit.
\end{abstract}

\section{Introduction}

With continuous development and progress of social economy, the living level of residents improves significantly. But the quality of citizens and especially physical quality of college students are not optimistic. The speed, endurance and explosive power of college students present different degrees of downtrend. The number of fat college students increases significantly, and students' physical quality is poor. Their conscious physical exercise awareness is insufficient. Thus, how to improve college students' physical quality becomes the problem that college physical education needs to solve. Physical exercise habit is the major force to support lasting and stable sports fitness belief. Sports fitness belief is formed by individuals on the basis of continuously deepening sports fitness awareness in sports practice process. It is a kind of persistence and assurance of physical exercise. Sports fitness belief will generate the motivation of physical exercise and facilitate students to persist in physical exercise.

\section{Analysis of essence and connotation of physical exercise habit}

Physical exercise habit refers to a behavioral pattern which is formed through repeated training in physical exercise process and can develop to become individuals' internal need. During judging individuals' physical exercise habit, weekly exercise times and time serve as the judgment indexes. If one takes exercise at least three times per week and more than 30min in each time, this indicates he (she) forms the physical exercise habit. On the contrary, he (she) lacks physical exercise habit. According to such definition, physical exercise habit mainly includes the following features. Firstly, individuals have clear value orientation for physical exercise events they persist in, and have distinct initiative and autonomy in physical exercise process; their active participation awareness is very high. Secondly, individuals have relatively fixed exercise contents and mode in physical exercise process. They can guarantee frequent exercise and persist in certain frequency of physical exercise per week. Finally, individuals can gain benignant feedback information in continuous physical exercise process, and their physical quality and spiritual awareness improve comprehensively. Besides, they can gain corresponding joyful experience. The formation of physical exercise habit makes physical exercise gradually become a relatively stable behavioral pattern in daily life. Individuals can engage in physical exercise all the time according to such mode. 


\section{Formation mechanism of physical exercise habit}

\section{Functions of sports and human internal needs}

Sports as a special social activity has multiple functions such as body building, entertainment and education. In the $21^{\text {st }}$ century, with social development and progress, the function and connotation of physical exercise gradually extend and expand. The fundamental functions of physical exercise are fitness and entertainment. Through proper exercise, individuals can improve athletic ability, metabolic capability, nervous centralis sensitivity, memory ability and observational ability, relieve physical and spiritual pressure and improve functions of brain and nervous system. Through proper physical exercise, athletic ability can boost, and cardiopulmonary respiratory function can be enhanced. The individuals with exercise habit have high immunity and resistance as well we excellent somatic functions. Besides, their living quality is good. Multiple forms of physical exercise events can entertain body and mind, improve interpersonal skills, reduce multiple pressures from life and work and improve living quality. Through certain form of physical exercise, people can break through and surpass themselves so as to achieve internal value pursuit.

\section{Formation and development of physical exercise motivation}

Motivation refers to inherent driving force to trigger and maintain an activity and make sure this activity can develop towards a goal. Physical exercise motivation has obvious directivity and target. It is the driving force which drives individuals to take exercise. The formation of physical exercise motivation involves multiple factors. The formation of physical exercise motivation is not just individuals' essential requirement for the purpose of building body, entertaining body and mind and improving living quality, but also the requirement of social progress for individuals. This process evolves from fuzziness to concreteness and from low grade to high grade. Firstly, individuals need physical exercise, which is the key factor to form physical exercise mechanism. They can build body, please the spirit, vent the pressure and train the will. Such internal needs promote lasting and stable physical exercise motivation. Secondly, living environment also has certain influence on individuals' physical exercise. The people around individuals have far-reaching influence on them. When one gains the joys of success due to exercise, individuals will see his (her) joys, inspiration and internal excitation. This will generate far-reaching influence on individuals. Finally, colleges and the society put forward requirements for individuals. Social requirements for talents are mainly reflected by various rules and regulations formulated by colleges. After graduates enter the society, different posts put forward high requirements for physical quality of talents. Graduates are required to have healthy body, good personality and active spirituality. Individuals' demand and employers' demand for talents can be satisfied by physical exercise, and can form internal driving force.

\section{Formation and development of individuals' physical exercise behavior}

The generation of individual exercise behavior is a key link to form exercise habit and also the direct driving force for individuals to generate exercise motivation. Only when there are external conditions can individuals' exercise behavior be generated smoothly. The key content is to maintain good exercise behavior from exercise behavior to finally formation of exercise habit. To make sure individuals keep such exercise behavior for a long time, individuals need to feel good effects of physical exercise in the specific physical exercise process. Individuals' participation in physical exercise is to better meet social employment, self-physiological and psychological needs. If these needs of individuals can be met continuously, they will form the habit of participating in physical exercise. But if one fails to reach the set purpose, individual physical exercise demand will gradually decline and even he (she) will give up the habit of physical exercise. Hence, only when an exercise effect is reached can individual physical exercise motivation goal be met. Meanwhile, individual exercise behavior can persist for a long time.

\section{Analysis of theoretical model of sports fitness belief}

Physical exercise habit is an organic system which is composed of sports fitness belief, physical exercise need and motivation as well as physical exercise behavior. In this system, fitness belief is the driving force which is relatively stable. In the system, external factors and personal factors such as 
weather situation, conditions of exercise place, work busyness, learning stress, sports facility condition and emotional state will promote or hinder the operation of the whole system. But as long as individual sports belief can persist, the whole system can operate normally, and individual physical exercise habit can be kept.

\section{Decomposition of theoretical model of sports fitness belief}

During studying physical exercise habit, we can understand sports fitness belief model as the felling aspects. Firstly, sports fitness belief further motivates individuals' physical exercise need so as to make sure individuals generate the motivation of physical exercise and persist in physical exercise behavior. Secondly, physical exercise behavior will react upon individuals' sports fitness belief. As such circulation goes on, individuals will finally form physical exercise habit. Thirdly, individuals' sports fitness cognition behavior is the foundation of forming sports fitness belief. All kinds of practice work in fitness process are the important basic conditions for individuals to engage in sports activities. Finally, individuals' personality trend to physical exercise is the condition of ensuring differences of sports activity. In exercise process, educational mechanism for individuals generated by sports plays a guiding role for individuals to engage in sports fitness activity. In our daily life, individuals' physical exercise behavior is often disturbed by all kinds of rejection factors. When physical exercise and rejection factors conflict, physical exercise is suppressed, and physical exercise behavior has to be abandoned. If it is not suppressed, physical exercise activity can be conducted.

\section{Analysis of forming process of sports fitness belief}

Sports fitness belief forms when individuals continuously cognize and experience physical exercise, which has promotion function for body and mind. Once sports fitness belief forms, it has persistence feature. After sports fitness belief forms, individual view is not changed easily. Such idea can make sure individuals pay attention to their health. In this way, physical exercise behavior and motivation can be motivated. Meanwhile, individuals can ensure long-time persistence in physical exercise and will not give up easily. The effect of individuals' physical exercise will act on sports fitness belief. Such cycle makes sure individuals' sports fitness belief and physical exercise behavior promote each other. When individuals persist in physical exercise for a long time, they can personally feel the positive effects of physical exercise on their body and mind, and the people around them make corresponding response to physical exercise. Gradually, individuals will form sports fitness self-cognition experience. Sports fitness self-cognition experience and positive evaluation of people around for physical exercise behavior facilitate formation and consolidation of individuals' sports fitness belief.

\section{Analysis of sports practice content}

There are diversified sports events. In the diversified sports system, the contents of sports practice is rich. Sports practice activities physical exercise teaching, physical exercise training, sports race and physical exercise. Meanwhile, sports practice activities cover various recreational activities, sports performance, sports enjoyment and sports tourism etc. Individuals can gain sports fitness cognition experience through participating in different sports events. Sports practice is not simply equal to physical exercise, but physical exercise is the basic sports practice activity which influences sports fitness belief. When individuals carry out physical exercise, the depth of exercise facilitates individuals to deepen their cognition degree for sports fitness. The depth of physical exercise refers to the initiative and enthusiasm for physical exercise as well as exercise time and energy in daily life. Usually, human physical exercise cognition experience will continuously deepen and develop as exercise goes deep.

\section{Analysis of individuals' tendency to physical exercise}

Individuals' tendency to physical exercise mainly includes individuals' interests in sports events, hobbies, needs, regions, the group engaging in such activity and their values etc. Individuals' tendency to physical exercise influences individuals' tendency to sports events and their choice. Meanwhile, this also influences the process of cognizing and experiencing sports fitness. Individuals generate keen interest in one sports event and hope to own healthy body and strong body through physical exercise, so they take more active part in physical exercise, continuously cognize and 
experience sports fitness so as to form correct sports fitness belief. Individuals' tendency to physical exercise is mainly restricted by local sports supporting factors and educational degree. Only when one region owns good physical education, sports venue and perfect sports service system can individuals' demand for physical exercise be promoted. In this way, individuals' sports interest and hobby can be trained. On the contrary, if sports cause in one region is weak, various sports facilities are not complete, and sports service capability is insufficient, individuals' enthusiasm for physical exercise will be greatly influenced, which is not beneficial to formation of sports fitness belief.

\section{Analysis of sports value education}

Sports value education mainly deepens and improves individuals' sports thought and idea through physical education, further enhances individuals' cognition for social status and functions of sports and deepens their understanding of promoting physical and psychological health through physical exercise. The tendency of sports value education individuals to physical exercise, sports practice and sports fitness cognition experience are important influence factors. The achievements of sports value education are influenced by the state, society and schools, followed by educational conditions and teaching level.

\section{References}

[1] Chen Lizhu, Bi Zhongchun, Theoretical analysis of factors influencing students' formation of physical exercise habit in stage of compulsory education, Journal of Beijing Sport University. 2006(03).

[2] Qiu Meiting, Jia Shaohua, Chen Qiongxia, Cai Ruiguang, Wang Liudong, Study on formation mechanism and influence factors of physical exercise habit, Journal of Capital Institute of Physical Education. 2005(06).

[3] Wang Rui, Wei Xiaofeng, Chi Mingli, On formation factors of college students' physical exercise habit, Journal of Northeast Agricultural University (Social Science Edition). 2009(04).

[4] Zhang Guiting, Jing Qiao, Study on factor system influencing college students’ physical exercise habit, Journal of Beijing Sport University. 2008(05).

[5] Li Caifeng, Analysis of factors influencing college students' physical exercise habit and improvement countermeasures, Journal of Chongqing Vocational\& Technical Institute. 2008(03).

[6] Li Lin, Ji Zhongqiu, Li Yanxia, Liu Weitong, Study of relevance of joint angle resetting method, minimum threshold measurement of exercise and strength reappearance method, Journal of Tianjin University of Sport. 2016(01).

[7] Fan Jinqin, Zhang Xiangqun, Fu Liming, Yang Ling, Fu Zuobing, Influence of different exercise moves on female college students with recessive obesity, Chinese Journal of School Health. 2016(03).

[8] Wang Jianzhou, Empirical study on influence of sports fitness scheme on college students' physical exercise attitude, Bulletin of Sport Science \& Technology. 2012(01). 